\title{
The Value of Shashen Maidong Decoction in the Treatment of Advanced Lung Cancer
}

\author{
Limei Qin* \\ Inner Mongolia Baicaotang Qin’s Zhong Meng Medical Hospital, Hohhot 010000, Inner Mongolia, China \\ *Corresponding author: Limei Qin, 18686057262@qq.com
}

\begin{abstract}
Objective: This study mainly explores the clinical effect of Shashen Maidong Decoction in the treatment of advanced lung cancer. Methods: Twelve patients with advanced lung cancer from Inner Mongolia Baicaotang Qin's Zhong Meng Medical Hospital were randomly selected from February 2016 to February 2021. They were divided into two groups: the reference group and study group by the digital table method. The reference group was treated with conventional chemotherapy while the study group was treated with Shashen Maidong Decoction plus conventional chemotherapy. Results: The remission rate of the study group was higher than that of the reference group, and the incidence of adverse reactions was lower than that of the reference group, $P<0.05$. The levels of $\mathrm{CD}_{3}{ }^{+}, \mathrm{CD}_{4}{ }^{+}$, and $\mathrm{CD}_{4}{ }^{+} / \mathrm{CD}_{8}{ }^{+}$in the study group were higher than those in the reference group $(P<0.05)$. After treatment, the quality of life score of the study group was higher than that of the reference group $(P<0.05)$. Conclusion: Shashen Maidong Decoction can effectively reduce adverse reactions and improve the condition of patients in the treatment of advanced lung cancer.
\end{abstract}

Keywords: Shashen Maidong Decoction; Advanced lung cancer; Clinical effect

Publication date: September 2021; Online publication: September 30, 2021

\section{Introduction}

Lung cancer is a very common tumor disease in clinical practice. Early accurate diagnosis and timely treatment of lung cancer play an extremely important role in the prognosis of patients. However, due to various factors, it is usually difficult to detect the disease in patients at an early stage. Usually, when the diagnosis is made, the disease has already progressed up to mid- or late stage; the best time for treatment has been delayed, thus increasing the difficulty of clinical treatment ${ }^{[1]}$. Especially in patients with advanced lung cancer, when their clinical symptoms gradually worsen and it is not possible to carry out surgical treatment, chemotherapy would be the only choice for disease control in order to prolong their survival time. However, in the process of radiotherapy, there would be adverse reactions, which would impact the patients' health and quality of life tremendously. According to relevant studies, the use of TCM syndrome differentiation while treating patients can improve their conditions to a certain extent. Based on this, this study explores the therapeutic effect of Shashen Maidong Decoction.

\section{Material and methods}

\subsection{Basic data}

In this study, 12 patients with advanced lung cancer from Inner Mongolia Baicaotang Qin's Zhong Meng Medical Hospital were randomly selected as the research subjects from February 2016 to February 2021. Clinical examination confirmed that the patients met the criteria for non-small cell lung cancer and the disease is in an advanced stage. The study was conducted with informed consent. The exclusion criteria 
were patients with abnormal liver and kidney function, as well as those with underlying diseases, such as anemia, diabetes, hyperthyroidism, and hypothyroidism. The patients were divided into two groups: the reference group and the study group with 6 patients in each group. There were 4 male patients and 2 female patients in the reference group, age ranging from 61 years old to 75 years old, with an average age of 69.31 \pm 1.25 years old. The shortest course of disease was 1 year while the longest was 3 years, with an average of $1.46 \pm 0.25$ years. On the other hand, there were 3 male patients and 3 female patients in the study group, age ranging from 59 years old to 76 years old, with an average age of $69.17 \pm 1.31$ years old. The shortest course of disease was 1 year while the longest was 4 years, with an average of $(1.51 \pm 0.27)$ years. There were three patients with stage IV lung cancer, two patients with stage III lung cancer, and one patient with stage II lung cancer. There was no significant difference between the two groups $(P>0.05)$.

\subsection{Methods}

The patients in the reference group were given gemcitabine hydrochloride on the first day and the eighth day; the dosage was $1250 \mathrm{mg} / \mathrm{m}^{2}$. They were also given $75 \mathrm{mg} / \mathrm{m}^{2}$ cisplatin during the first three days, which lasted for 60 days.

The study group was treated the same as the reference group but with the addition of Shashen Maidong Decoction: $30 \mathrm{~g}$ of Astragalus membranaceus, Hedyotis diffusa, and Lycoris chinensis each; $10 \mathrm{~g}$ of trichosanthin, Ophiopogon japonicus, Radix Glehniae, Polygonatum odoratum, hawthorn, and mulberry leaves each; $6 \mathrm{~g}$ of liquorice. With cough symptoms, Platycodon grandiflorum and Fritillaria thunbergii were added. Patients with pleural effusion were given additional Poria cocos and Tinglizi while Rhizoma corydalis and Rhizoma Curcumae were added when patients presented with chest pain. All of it were decocted with water and $300 \mathrm{ml}$ of the decoction was taken by the patients in the morning and evening for 60 days.

\subsection{Evaluation index}

The clinical efficacy was evaluated strictly according to the standard of diagnosis and treatment of lung cancer, which was divided into complete remission, partial remission, stable, and progress. The effective remission rate (effective treatment rate $)=($ complete remission + partial remission $) /$ total cases $\times 100 \%{ }^{[2]}$. Adverse reactions such as leucopenia, anemia, dizziness, and vomiting were also observed.

In addition, the serum immunological indices, $\mathrm{CD}_{3}{ }^{+}, \mathrm{CD}_{4}{ }^{+}$, and $\mathrm{CD}_{4}{ }^{+} / \mathrm{CD}_{8}{ }^{+}$, of the two groups were detected. Visual Analogue Scale (VAS) was used to evaluate the improvement of pain with a total score of 10 points; the lower the score, the less painful it was. SF-36 (36-Item Short Form Survey) was used to evaluate the improvement of patients' quality of life, including physiological function, mental health, and psychiatric health, with a total score of 100 points. The higher the score, the higher the quality of life ${ }^{[3]}$.

\subsection{Statistical analysis}

Statistical Package for the Social Sciences (SPSS) version 23.0 was used for analysis, $t$ and $X^{2}$ were used for test, and $(\bar{x} \pm \mathrm{s})(\mathrm{n} / \%)$ was used for expression. If $P<0.05$, it was considered that there is a difference in data.

\section{Results}

\subsection{Clinical efficacy}

According to the statistics in Table 1, the effective rate of the study group was higher than that of the reference group, $P<0.05$. 
Table 1. Efficacy statistics (n /\%)

\begin{tabular}{lcccccc}
\hline Group & N (example) & Complete remission & Partial remission & Stable & Progress & Total effective rate \\
\hline Reference group & 6 & 1 & 3 & 2 & 0 & 66.66 \\
Study group & 6 & 3 & 3 & 0 & 0 & 100.00 \\
$X^{2}$ & & & & & & 4.1275 \\
$P$ & & & & & & 0.0497 \\
\hline
\end{tabular}

\subsection{Serum immunological indices}

According to the data statistics in Table 2, the serum immunological indices of the study group were higher than those of the reference group, $P<0.05$.

Table 2. Statistics of serum immunological indices after treatment $(\bar{x} \pm \mathrm{s})$

\begin{tabular}{lccccc}
\hline Group & $\mathrm{N}($ example $)$ & $\mathrm{CD}_{3}{ }^{+}$ & $\mathrm{CD}_{4}{ }^{+}$ & $\mathrm{CD}_{8}{ }^{+}$ & $\mathrm{CD}_{4}{ }^{+} / \mathrm{CD}_{8}{ }^{+}$ \\
\hline Reference group & 6 & $70.15 \pm 3.14$ & $42.12 \pm 2.57$ & $35.47 \pm 3.15$ & $1.81 \pm 0.29$ \\
Study group & 6 & $75.33 \pm 4.32$ & $46.52 \pm 3.12$ & $40.01 \pm 3.69$ & $2.37 \pm 0.48$ \\
$t$ & & 2.3758 & 2.6663 & 2.2921 & 2.4460 \\
$P$ & & 0.0389 & 0.0236 & 0.0449 & 0.0345 \\
\hline
\end{tabular}

\subsection{Pain and improvement of quality of life}

There was no significant difference between the two groups before treatment $(P>0.05)$. However, after treatment, the VAS score of the study group was lower than that of the reference group and the SF-36 score was higher than that of the reference group, $P<0.05$ as shown in Table 3.

Table 3. Statistics of VAS score and SF-36 score $(\bar{x} \pm s)$

\begin{tabular}{lccccc}
\hline \multirow{2}{*}{ Group } & $\mathrm{N}$ & \multicolumn{2}{c}{ VAS score } & \multicolumn{2}{c}{ SF-36 score } \\
\cline { 2 - 5 } & (example) & Before treatment & After treatment & Before treatment & After treatment \\
\hline Reference group & 6 & $6.14 \pm 1.35$ & $4.57 \pm 1.07$ & $71.33 \pm 3.67$ & $83.31 \pm 4.25$ \\
Study group & 6 & $6.21 \pm 1.42$ & $3.12 \pm 1.02$ & $72.41 \pm 4.52$ & $89.34 \pm 3.51$ \\
$t$ & & 0.0875 & 2.4026 & 0.4544 & 2.6797 \\
$P$ & & 0.9320 & 0.0371 & 0.6593 & 0.0231 \\
\hline
\end{tabular}

\subsection{Adverse reactions}

The incidence of adverse reactions in the study group was lower than that in the reference group, $P<0.05$ (Table 4).

Table 4. Incidence of adverse reactions (n /\%)

\begin{tabular}{lccccc}
\hline Group & N (example) & Dizziness and vomiting & Leukopenia & Anemia & Total incidence \\
\hline Reference group & 6 & 2 & 0 & 1 & 50.00 \\
Study group & 6 & 1 & 0 & 0 & 16.66 \\
$X^{2}$ & & & & & 4.0157 \\
$P$ & & & & & 0.0495 \\
\hline
\end{tabular}




\section{Discussion}

At present, lung cancer is a common malignant tumor disease in clinical practice. The occurrence of lung cancer has a certain relationship with smoking or long-term inhalation of harmful gases in daily life. Once lung cancer occurs, patients will develop mild symptoms at the early stage. With the progression of the disease, cough, chest tightness, and fever gradually appear, especially during the late stages. At this point of time, clinical treatment becomes more difficult as the best time for surgical treatment is missed, thus only chemotherapy can be used for treatment. However, chemotherapy leads to a series of adverse reactions, directly affecting the patients' quality of life ${ }^{[4]}$.

From the perspective of traditional Chinese medicine, lung cancer is included in the category of "hemoptysis" and "lung accumulation." Traditional Chinese medicine generally believes that lung cancer is caused by the invasion of external pathogens, such as phlegm, dampness, and toxin, which lead to Qi deficiency and imbalance of yin and yang, resulting in the loss of lung circulation and blood circulation. Eventually, these lead to lung accumulation, thus resulting in the disease. Therefore, the main principles of treatment in traditional Chinese medicine include nourishing yin, tonifying the lung, clearing heat, and replenishing qi, where the use of Shashen Maidong Decoction aids in the achievement of significant results

${ }^{[5]}$. Yanhusuo in Shashen Maidong Decoction promotes blood circulation along with Qi circulation while relieving pain; Polygonatum odoratum and trichosanthin have the effect of promoting body fluid while mulberry leaves diffuse dryness-heat with lightness; Radix Glehniae and Radix Ophiopogonis nourish the lung and stomach; Hedyotis diffusa clears heat, detoxifies, and relieves pain; Glycyrrhiza neutralizes the drug properties. The combination of many drugs plays a therapeutic effect of detoxification, clearing away heat, nourishing yin, and moistening the lung.

In this study, the treatment efficiency, immune function, and quality of life of the study group were higher than those of the reference group, with significant differences between the two groups. This shows that Shashen Maidong Decoction can effectively improve pain and the quality of life of patients with advanced lung cancer. This is mainly because the traditional Chinese medicine focuses on freeing collateral vessels and tonifying the middle energizer. Therefore, it plays the role of clearing away heat, resolving phlegm, detoxifying, replenishing qi, and inhibiting the development of the tumor to a certain extent ${ }^{[6-9]}$.

In conclusion, the clinical effect of Shashen Maidong Decoction in the treatment of advanced lung cancer is extremely significant in reducing pain and improving patients' quality of life.

\section{Disclosure statement}

The author declares that there is no conflict of interest.

\section{References}

[1] Wang G, Zhu X, Gu L, et al., 2019, Clinical Study of Modified Shashen Maidong Decoction Combined with Etoposide and Cisplatin in the Treatment of Small Cell Lung Cancer. Chinese and Foreign Medical, 38(32): 178-180.

[2] Li Q, Zhang W, 2020, Effect of Modified Shashen Maidong Decoction Combined with Chemotherapy in the Treatment of Advanced Non-Small Cell Lung Cancer. Medical Dietotherapy and Health, 18(007): 35-35, 37.

[3] $\mathrm{Hu}$ P, 2020, Effect of Modified Shashen Maidong Decoction Combined with Chemotherapy on CD4+ and CD8+ in Patients with Advanced Lung Cancer. Journal of Modern Medicine and Health Research, 57(02): 79-80. 
[4] Xia X, Zheng Z, 2020, Clinical Effect Analysis of Modified Shashen Maidong Decoction and Chemotherapy in the Treatment of Advanced Non-Small Cell Lung Cancer. Electronic Journal of Clinical Medicine Literature, 453(32): 157, 169.

[5] Su T, Wang X, Li C, et al., 2019, An Ethanolic Extract of Bailian (Radix Ampelopsis Japonicae): Demonstration of Colorectal Cancer Treatment Efficacy Via Inhibition of B-Catenin Signaling In Vitro. Journal of Traditional Chinese Medicine, 39(3): 59-65.

[6] $\mathrm{Hu}$ P, 2020, Effect of Modified Shashen Maidong Decoction Combined with Chemotherapy on CD4+ CD8+ in Patients with Advanced Lung Cancer. Modern Medicine and Health Research (electronic version), 4(2): 75-76.

[7] Lou D, 2019, Effect of Modified Shashen Maidong Decoction Combined with Chemotherapy on Improving Immune Function of Lung Cancer Patients. Journal of Taishan Medical College, 040(010): 769-770.

[8] Liu C, Luo X, Liu H, 2020, Effect of Modified Shashen Maidong Decoction Combined with Gefitinib on Immune Function and Adverse Reactions in Elderly Patients with Advanced Non-Small Cell Lung Cancer. Shaanxi Traditional Chinese Medicine, (2): 183-186.

[9] Li N, Zhong S, 2020, Buzhong Yiqi Decoction and Shashen Maidong Decoction Combined with Chemotherapy in the Treatment of Non-Small Cell Lung Cancer and Its Influence on Tumor Treatment Effect, Quality of Life, Drug Toxicity and Curative Effect of Traditional Chinese Medicine. Heilongjiang Traditional Chinese Medicine, 309(1): 110-111. 